Pacific Journal of Mathematics

RESIDUAL PROPERTIES OF FREE GROUPS 


\title{
RESIDUAL PROPERTIES OF FREE GROUPS
}

\author{
STEPHen J. PRIDE
}

In this paper the following theorem is proved: if $\pi$ is an infinite set of primes and $n$ is an odd integer greater than one, then free groups are residually $\{P S L(n, p) ; p \in \pi\}$. As a by-product of the proof new generators of $S L(n, p)$ are obtained for nearly all primes $p$.

1. The main result. For unexplained notation the reader is referred to [8].

Let $\mathscr{A}_{1}$ and $\mathscr{A}_{2}$ be sets of groups. $\mathscr{A}_{1}$ is said to be residually $\mathscr{A}_{2}$ iff, for each group $G$ belonging to $\mathscr{A}_{1}$ and each non-identity element $g$ of $G$ there is a homomorphism $\varphi$ (depending on $G$ and $g$ ) which maps $G$ onto some element $H$ of $\mathscr{A}_{2}$, and is such that $\varphi(g)$ is not the identity of $H$. An equivalent formulation is: for each $G$ in $\mathscr{A}_{1}$ there is a set of normal subgroups $\left\{N_{i}\right\}_{i \in I}$ of $G$ such that $\bigcap_{i \in I} N_{i}=1$ and for each $i$ in $I, G / N_{i}$ is isomorphic to an element of $\mathscr{A}_{2}$. It is obvious that if $\mathscr{A}_{1}$ and $\mathscr{A}_{2}$ are sets of groups and some or all of the members of $\mathscr{A}_{1}$ and $\mathscr{A}_{2}$ are replaced by isomorphic copies, yielding new sets $\mathscr{A}_{1}^{\prime}$ and $\mathscr{A}_{2}^{\prime}$ respectively, then $\mathscr{A}_{1}$ is residually $\mathscr{A}_{2}$ iff $\mathscr{A}_{1}^{\prime}$ is residually $\mathscr{A}_{2}^{\prime}$. It is also easy to see that if $\mathscr{A}_{1}$ is residually $\mathscr{A}_{2}$, and $\mathscr{A}_{2}$ is residually $\mathscr{A}_{3}$, then $\mathscr{A}_{1}$ is residually $\mathscr{A}_{3}$.

Let $\left\{x_{1}, x_{2}, x_{3}, \cdots\right\}$ be a fixed but arbitrary countably infinite set, and let $F_{n}$ be the free group freely generated by $\left\{x_{1}, x_{2}, \cdots, x_{n}\right\}$. Denote by $\mathscr{F}$ the set $\left\{F_{n}: n \geqq 2\right\}$. In recent years there has been some investigation into which sets, $\mathscr{A}$, of groups are such that $\mathscr{F}$ is residually $\mathscr{A}$. The two-generator groups in $\mathscr{A}$ must of necessity generate the variety, $\mathcal{O}$, of all groups. It has been conjectured by $S$. Meskin that this condition is also sufficient. A rich source of sets of groups which generate $O$ is a result of Heineken and Neumann [3] which states that every infinite set of pairwise non-isomorphic known (1967) finite non-abelian simple groups generates the variety of all groups. This theorem has presumably inspired several of the results obtained so far. Thus Katz and Magnus [5] have proved that $\mathscr{F}$ is residually $\left\{A_{n}: n \in J\right\}$, where $A_{n}$ is the alternating group on $\{1,2, \cdots, n\}$ and $J$ is an infinite set of positive odd integers; and Gorcakov and Leveuk [2] have proved that $\mathscr{F}$ is residually any infinite subset of the set of simple groups $P S L\left(2, p^{r}\right)$. This latter result generalizes theorems obtained in [6], [5] and [7], which consider the cases $r=1$ and $p$ variable, $r>1$ and fixed and $p$ variable, $p>11$ and fixed and $r$ variable, respectively.

In this paper the following main result is obtained. 
THEOREM 1. Let $n$ be an odd integer greater than one, and let $\pi$ be an infinite set of primes. Then $\mathscr{F}$ is residually $\{P S L(n, p)$ : $p \in \pi\}$.

Before discussing the proof of Theorem 1 some notation and definitions will be introduced. Let $R$ be a commutative ring with identity 1 . The ring of polynomials in the indeterminant $x$ with coefficients from $R$ will be denoted by $R[x]$. The degree of an element $f(x)$ of $R[x]$ will be written as $\operatorname{deg}(f(x))$. As is well-known (see [4], page 56) the $n \times n$ matrices with entries from $R$ form a ring with identity. The identity will be denoted by $E$. The $n \times n$ matrix with 1 in its $i$ th row and $j$ th column and zeros elsewhere will be denoted by $E_{i j}(i, j=1,2, \cdots, n)$, and $E_{(n+i) j}, E_{(n+i)(n+j)}, E_{i(n+j)}(i, j=1,2, \cdots$, $n$ ) will all be defined to be equal to $E_{i j}$. The multiplicative semigroup of the ring of $n \times n$ matrices with entries from $R$ has a subsemigroup consisting of all matrices which have a single nonzero entry, namely 1 , in each row and each column. This sub-semigroup is in fact a group, isomorphic to the symmetric group on $\{1,2, \cdots, n\}$. An isomorphism is given by:

$$
\sigma \longrightarrow \sum_{i=1}^{n} E_{i \sigma(i)},
$$

where $\sigma$ is a permutation of $\{1,2, \cdots, n\}$. The matrix $\sum_{i=1}^{n} E_{i \sigma(i)}$ will be called the permutation matrix corresponding to $\sigma$. When no confusion can arise, and if it is convenient to do so, the matrix $\sum_{i=1}^{n} E_{i \sigma(i)}$ will be denoted by the permutation $\sigma$.

For the rest of this section $n$ will denote a fixed but arbitrary odd integer greater than one, and $p$ (possibly subscripted) will stand for a prime number. To simplify the proof of Theorem 1, use is made of the following two results:

(i) $\mathscr{F}$ is residually $\left\{F_{2}\right\}$,

(ii) For each $k \geqq 2,\left\{F_{2}\right\}$ is residually $\left\{T_{k}\right\}$, where $T_{k}=\left(a, b \mid a^{k}\right)$. The former result is proved in [6], whilst Lemma 1 of [5] proves (ii) for the case $k=2$, and the proof for $k>2$ is entirely analogous. Using (i) and (ii) reduces the proof of Theorem 1 to showing that $\left\{T_{n}\right\}$ is residually $\{P S L(n, p): p \in \pi\}$.

The first step in proving that $\left\{T_{n}\right\}$ is residually $\{P S L(n, p): p \in \pi\}$ is to find a group of $n \times n$ matrices which is isomorphic to $T_{n}$. Consider the ring of $n \times n$ matrices with entries from $Z[x]$. The multiplicative semigroup of this ring has a sub-semigroup consisting of all matrices with determinant \pm 1 . This sub-semigroup is a group, called the group of units. The permutation matrix $X$ corresponding to the permutation $(1,2,3, \cdots, n)$, and the matrix $Y=E+x \sum_{j=2}^{n} E_{j 1}$ are in the group of units. They therefore generate a subgroup, $\mathscr{U}_{n}$, 
of this group. Notice that in this group $X$ has order $n$ and $Y$ has infinite order. In $\S 2$ the following result is proved.

LEMMA 1. When a product of the form

$(*)$

$$
Y^{\nu} X^{\delta_{1}} Y^{m_{1}} \cdots X^{\delta_{r}} Y^{m_{r}} X^{\mu}
$$

-where $r \geqq 0$, the $\delta_{i}$ can have the values $1,2, \cdots, n-1$, the $m_{i}$ can have any integer values except zero, $\nu$ can have any integer value, $\mu$ can be $0,1,2, \cdots, n-1, \nu$ and $\mu$ cannot be zero simultaneously unless $r \geqq 1$-is multiplied out, it has an entry of degree at least one, provided $\nu$ and $r$ are not both zero.

From this lemma follows immediately

THEOREM 2. $\mathscr{C}_{n}$ and $T_{n}$ are isomorphic.

The problem is now reduced to showing that $\left\{\mathscr{C}_{n}\right\}$ is residually $\{P S L(n, p): p \in \pi\}$. There are plenty of homomorphisms from $\mathscr{C}_{n}$ into $S L(n, p)$. In fact, let $\alpha$ be a nonzero element of $G F(p)$. Then, by Theorem 4 of Chapter III [4], there is a ring homomorphism of $Z[x]$ onto $G F(p)$ which maps $x$ to $\alpha$. This homomorphism induces a homomorphism $\varphi_{\alpha}$ from the multiplicative semigroup of all $n \times n$ matrices with entries from $Z[x]$ to the multiplicative semigroup of all $n \times n$ matrices with entries from $G F(p)$. The value of $\varphi_{\alpha}$ at the matrix $M$ is obtained by replacing all appearances of $x$ in $M$ by $\alpha$, and replacing all integers appearing as coefficients in the polynomials in $M$ by their congruence classes modulo the prime $p$. When restricted to $\mathscr{U}_{n}, \varphi_{\alpha}$ is a group homomorphism with range contained in $S L(n, p)$. Let $\varphi_{\alpha}(X)=C$ and $\varphi_{\alpha}(Y)=D(\alpha)$. It is easy to see that the subgroup of $S L(n, p)$ generated by $C$ and $D(\alpha)$ is the same as that generated by $C$ and $D=D(1)$. For there are integers $t$ and $u$ such that $t \alpha=1$ and $u 1=\alpha$, and so $D(\alpha)^{t}=D$ and $D^{u}=D(\alpha)$. In $\S 3$ the following result is proved.

Theorem 3. Let $p$ be a prime which does not divide $3(n-1)$. Then $C$ and $D$ generate $S L(n, p)$.

(If $p$ divides $3(n-1)$, the validity of the theorem remains undecided.)

It follows immediately from Theorem 3 that $\varphi_{\alpha}$ is a homomorphism of $\mathscr{U}_{n}$ onto $S L(n, p)$ for all but a finite number of primes $p$.

Using Lemma 1 and Theorems 2 and 3, it is now possible to prove that $\left\{\mathscr{C}_{n}\right\}$ is residually $\{P S L(n, p): p \in \pi\}$. It is well-known (see [8], 
page 158) that the centre of $S L(n, p)$ consists of all scalar matrices $\lambda E$, where $\lambda^{n}=1$. Given a non-identity element $W$ of $\mathscr{C}_{n}$, it will be shown that there is a prime $p$ in $\pi$, and a homomorphism $\varphi$ of $\mathscr{U}_{n}$ onto $S L(n, p)$ such that $\varphi(W)$ does not belong to the centre of $S L(n, p)$. Then the composition of $\varphi$ with the natural homomorphism of $S L(n, p)$ onto $\operatorname{PSL}(n, p)$ gives a homomorphism of $\mathscr{C}_{n}$ onto $P S L(n, p)$ which does not map $W$ to the identity.

Thus, let $W$ be a non-identity element of $\mathscr{U}_{n}$. Then $W$ can be expressed uniquely as a product of the form $\left({ }^{*}\right)$ (see Lemma 1). First suppose that in the product $\left(^{*}\right) \nu=0$ and $r=0$, so that $W=X^{\mu}$, where $\mu$ is an integer and $0<\mu<n$. Let $p_{0}$ be a prime in $\pi$ which does not divide $3(n-1)$. Then the homomorphism of $\mathscr{C}_{n}$ onto $S L\left(n, p_{0}\right)$ determined by

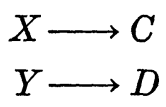

does not map $W$ to the centre of $S L\left(n, p_{0}\right)$.

Suppose now that the product $\left(^{*}\right)$ is such that not both of $\nu$ and $r$ are zero. Then by Lemma $1, W$ has an entry

$$
a_{0}+a_{1} x+\cdots+a_{s} x^{s} \text { with } a_{s} \neq 0, s \geqq 1 \text {. }
$$

Let $p_{0}$ be a prime in $\pi$ with the property

$$
p_{0}-1>\max \left\{\left|a_{s}\right|, s(n+1)\right\} \text {. }
$$

The congruence class of an integer $k \bmod p_{0}$ will be denoted by $\bar{k}$. Consider the polynomials

$$
\begin{aligned}
& f(x)=\bar{a}_{0}+\bar{a}_{1} x+\cdots+\bar{a}_{s} x^{s}, \\
& g(x)=f(x)\left[(f(x))^{n}-\overline{1}\right],
\end{aligned}
$$

which are elements of $G F\left(p_{0}\right)[x]$. Since $\bar{a}_{\varepsilon} \neq \overline{0}, \operatorname{deg}(f(x))=s$, and so deg $(g(x))=s(n+1)$. By the choice of $p_{0}$ there is a nonzero element $\alpha$ of $G F\left(p_{0}\right)$ which is not a root of $g(x)$.

Let $\phi$ be the homomorphism of $\mathscr{L}_{n}$ onto $S L\left(n, p_{0}\right)$ determined by

$$
\begin{aligned}
& X \longrightarrow C \\
& Y \longrightarrow D(\alpha) .
\end{aligned}
$$

(Note that $p_{0}$ does not divide $3(n-1)$, so Theorem 3 applies.) The entries of $\varphi(W)$ are obtained from those of $W$ by replacing $x$ by $\alpha$ and working $\bmod p_{0}$. Hence $\varphi(W)$ has

$$
f(\alpha)=\bar{a}_{0}+\bar{a}_{1} \alpha+\cdots+\bar{a}_{s} \alpha^{s}
$$

as one of its entries. By the choice of $\alpha, f(\alpha) \neq \overline{0}$ and $f(\alpha)^{n} \neq \overline{1}$, so 
clearly $\varphi(W)$ does not lie in the centre of $S L\left(n, p_{0}\right)$.

2. Proof of Lemma 1. In this and the next section it will be useful to keep in mind the following rule for calculating with permutation matrices. If $M$ is a $u \times u$ matrix and $P$ is the permutation matrix corresponding to a permutation $\sigma$ of $\{1,2, \cdots, u\}$, then $P M$ is obtained from $M$ by replacing row $i$ by row $\sigma(i)$, and $M P$ is obtained from $M$ by replacing column $i$ by column $\sigma^{-1}(i)(1 \leqq i \leqq u)$.

Before proving Lemma 1 , it should be pointed out that the result is also valid when $n$ is even (the proof given below does not depend upon $n$ being odd), but in this case the permutation matrix corresponding to $(1,2,3, \cdots, n)$ has determinant -1 , so that the result is not of any use here.

A product of the form $\left(^{*}\right.$ ) (as in the statement of Lemma 1) in which $\nu=\mu=0$ will be called a product of type- $(X Y)$. When such a product is multiplied out, a matrix with entries $\xi_{i j}^{(r)}(i, j=1,2, \cdots$, $n$ ) from $Z[x]$ is obtained. The following assertion will be proved by induction on $r$.

$$
\begin{array}{ll}
\operatorname{deg}\left(\xi_{11}^{(r)}\right) & =r \\
\operatorname{deg}\left(\xi_{1 j}^{(r)}\right) & <r \text { for } j=2,3, \cdots, n .
\end{array}
$$

For $r=1$ the product is just $X^{\delta_{1}} Y^{m_{1}}$, which is equal to $X^{\delta_{1}}+$ $m_{1} x \sum_{j=2}^{n} E_{\left(n+j-\delta_{1}\right) 1}$. Thus

$$
\xi_{i 1}^{(1)}=\left\{\begin{array}{ll}
m_{1} x & i \neq n+1-\delta_{1} \\
1 & i=n+1-\delta_{1}
\end{array} .\right.
$$

All other entries of $X^{\delta_{1}} Y^{m_{1}}$ are either zero or one. Since $0<\delta_{1}<n$, it follows that $1<n+1-\delta_{1}<n+1$, so that $\xi_{11}^{(1)}$ is $m_{1} x$. Thus $(++)$ holds when $r=1$.

Now assume $(++)$ holds for all $s<r$, where $r>1$. The first row of $X^{\delta_{1}} Y^{m_{1}} \ldots X^{\delta_{r-1}} Y^{m_{r-1}} X^{\delta_{r}} Y^{m_{r}}$ is obtained from that of $X^{\delta_{1}} Y^{m_{1}} \ldots$ $X^{\delta_{r-1}} Y^{m_{r-1}}$ by right multiplication by $X^{\delta_{r}} Y^{m_{r}}$. Thus

$$
\xi_{11}^{(r)}=\sum_{\substack{1 \leq j \leq n \\ j \neq n+1-\delta_{r}}} m_{r} x \xi_{1 j}^{(r-1)}+\xi_{1\left(n+1-\delta_{r}\right)}^{(r-1)} .
$$

Since $1<n+1-\delta_{r}<n+1$, it follows that

$$
\begin{aligned}
\operatorname{deg}\left(\xi_{11}^{(r)}\right) & =\operatorname{deg}\left(\xi_{11}^{(r-1)}\right)+1 \\
& =r .
\end{aligned}
$$

Now except for column one, every column of $X^{\delta_{r}} Y^{m_{r}}$ contains only zeros and ones. Hence for $2 \leqq j \leqq n$, 


$$
\begin{aligned}
\operatorname{deg}\left(\xi_{1 j}^{(r)}\right) & \leqq \max \left\{\operatorname{deg}\left(\xi_{1 t}^{(r-1}\right): t=1,2, \cdots, n\right\} \\
& \leqq r-1 \\
& <r .
\end{aligned}
$$

This shows that $(++)$ holds for $r$, and completes the induction proof.

Now take a product of the general form $\left({ }^{*}\right)$ in which not both of $\nu$ and $r$ are zero, and let $W$ be the matrix obtained when this product is multiplied out. It is required to show that $W$ has an entry of degree at least one.

Case (i). $\nu=\mu=0$. The product is of type- $(X Y)$, so $W$ has an entry of degree $r$, by $(++)$.

Case (ii). $\nu \neq 0, \mu \neq 0$. Since

$$
W^{-1}=X^{n-\mu} Y^{-m_{r}} X^{n-\delta} r \ldots Y^{-m_{1}} X^{n-\hat{o}_{1}} Y^{-\nu}
$$

and the product on the right is of type- $(X Y), W^{-1}$ has an entry of degree at least one by $(++)$; consequently $W$ has also.

Case (iii). $\nu \neq 0, \mu=0$. If $r=0, W$ is just $Y^{\nu}$, which has $\nu x$ as one of its entries. Suppose then that $r \geqq 1$. $X^{\delta_{1}} Y^{m_{1}} \cdots X^{\delta_{r}} Y^{m_{r}}$ is a product of type- $(X Y)$, so the entries $\xi_{1 j}^{(r)}(j=1,2, \cdots, n)$ in the first row of the matrix $U$ obtained when this product is multiplied out satisfy $(++)$. The first row of $W$ is the same as that of $U$, so $W$ has an entry of degree $r$.

Case (iv). $\nu=0, \mu \neq 0$. If $U$ is the matrix obtained when $X^{\delta_{1}} Y^{m_{1}} \cdots X^{\delta_{r}} Y^{m_{r}}$ is multiplied out, then $U$ has an entry of degree $r$, and since $W$ is just obtained from $U$ by a permutation of columns, $W$ also has an entry of degree $r$.

This completes the proof of Lemma 1.

3. Proof of Theorem 3. The following definitions are used. A matrix of the form $E+\lambda E_{i j}$, where $\lambda \neq 0$ and $i \neq j$, will be called a transvection. In a group $G$ the commutator $\left[g_{1}\right]$ of $g_{1} \in G$ will be defined to be $g_{1}$, the commutator $\left[g_{1}, g_{2}\right]$ of $g_{1}, g_{2} \in G$ will be defined to be $g_{1} g_{2} g_{1}^{-1} g_{2}^{-1}$, and for $n \geqq 3,\left[g_{1}, g_{2}, \cdots, g_{n}\right]$ will be defined to be [ $\left.\left[g_{1}, \cdots, g_{n-1}\right], g_{n}\right]$. If $S$ is a nonempty subset of $G$ then $\operatorname{sgp} S$ will denote the subgroup of $G$ generated by $S$.

Let $n$ denote a fixed but arbitrary odd integer greater than one, and let $p$ be a fixed but arbitrary prime which does not divide $3 n-3$. It is required to show that the elements

$$
C=\sum_{i=1}^{n} E_{i(i+1)}
$$




$$
D=E+\sum_{j=2}^{n} E_{j 1}
$$

of $S L(n, p)$ generate this group. It will be shown below that the transvection $E+E_{1 n}$ belongs to $\operatorname{sgp}\{C, D\}$, and from this the result follows, as is now indicated.

It is well-known (see [8], page 158) that the transvections

$$
E+\lambda E_{i j}(i \neq j ; i, j=1,2, \cdots, n),
$$

where $\lambda$ ranges over the nonzero elements of $G F(p)$, generate $S L(n, p)$. In fact, it is enough to choose one value of $\lambda$, say $\lambda_{i j}$, for each pair $(i, j)$. For $\lambda_{i j}$ has order $p$ in the additive group of $G F(p)$, and so as $t$ runs through the integers from 1 to $p-1, t \lambda_{i j}$ assumes every nonzero element of $G F(p)$. Since

$$
\left(E+\lambda_{i j} E_{i j}\right)^{t}=E+\left(t \lambda_{i j}\right) E_{i j}(i \neq j ; i, j=1,2, \cdots, n)
$$

all transvections can be obtained from the $E+\lambda_{i j} E_{i j}$. Notice that, in particular, the value 1 can be chosen for each $\lambda_{i j}$.

Let $\mathscr{C}=\operatorname{sgp}\left\{E+E_{1 n}, C\right\}$. Now for $i, j=1, \cdots, n$

$$
C E_{i j} C^{-1}=E_{(n+i-1)(n+j-1)} \text {. }
$$

Therefore

$$
\begin{aligned}
C^{r}\left(E+E_{1 n}\right) C^{-r} & =E+E_{(n+1-r)(n-r)} \\
& =\tau_{r}, \text { say }(0 \leqq r \leqq n-1) .
\end{aligned}
$$

It is easily shown that

$$
\left[\tau_{0}, \tau_{1}, \cdots, \tau_{s}\right]=E+E_{1(n-s)}(0 \leqq s \leqq n-2) .
$$

Thus $\mathscr{E}$ contains all the transvections

$$
E+E_{1 h} h=2,3, \cdots, n .
$$

Finally, using $\left(^{* *}\right) k$ times $(0 \leqq k \leqq n-1)$ gives

$$
C^{k}\left(E+E_{1 h}\right) C^{-k}=E+E_{(n+1-k)(n+h-k)}, h=2,3, \cdots, n,
$$

and so $\mathscr{H}$ contains all the transvections

$$
E+E_{i j}(i \neq j ; i, j=1,2, \cdots, n) .
$$

Therefore $\mathscr{H}=S L(n, p)$.

It will now be shown that $E+E_{1 n}$ belongs to $\operatorname{sg} p\{C, D\}$. Straightforward computations show 


$$
\begin{aligned}
{\left[D^{-1}, C^{-1}\right] D } & =E+E_{11}+E_{12}-E_{21}-E_{22} \\
& =P, \text { say } \\
{\left[D^{-1}, C^{-2}\right] D } & =E+E_{11}+E_{13}-E_{31}-E_{33} \\
& =Q, \text { say } \\
C^{-1}\left(\left[D^{-1}, C^{-1}\right] D\right) & =E+E_{22}+E_{23}-E_{32}-E_{33} \\
& =R, \text { say. }
\end{aligned}
$$

Let $t$ be an integer such that $6 t \equiv 1 \bmod p$ (such a $t$ exists since $p$ is not 2 or 3 ). Then

$$
\left(Q P^{-1} R^{-1}\right)^{2 t}=E-E_{13}+E_{23} .
$$

This element will be denoted by $T$. It turns out to be extremely useful.

Another useful element is

$$
T^{2} R P=\sum_{i=4}^{n} E_{i i}+E_{12}+E_{23}+E_{31} .
$$

This is just the permutation matrix corresponding to the permutation (123). Since, for $m \geqq 3$ and odd, the permutations (123) and $(123 \cdots m)$ generate the alternating group $A_{m}$ ([1], page 67), it follows that $\operatorname{sgp}\{C, D\}$ contains all even permutation matrices.

Suppose that $n$ is greater than 3. It is easy to see that

$$
\begin{gathered}
(34 \cdots n) T^{-1}(34 \cdots n)^{-1}=E+E_{1 n}-E_{2 n} \\
(1 s)(2, s+1)\left(E+E_{1 n}-E_{2 n}\right)(1 s)(2, s+1)=E+E_{s n}-E_{(s+1) n} \\
\quad(3 \leqq s \leqq n-2)
\end{gathered}
$$

and

$$
(123)^{-1}\left(E+E_{1 n}-E_{2 n}\right)(123)=E+E_{2 n}-E_{3 n} .
$$

From (1), (2) and (3) it follows that $\operatorname{sgp}\{C, D\}$ contains all the matrices

$$
\Lambda_{\lambda}=E+E_{\lambda n}-E_{(\lambda+1) n} \quad 1 \leqq \lambda \leqq n-2 .
$$

This is also obviously true if $n$ equals 3 .

Now take the matrix

$$
C D C^{-1}=E+\sum_{i=1}^{n-1} E_{i n} .
$$

Multiplying by $A_{n \rightarrow 2}$ (on either side, since each $\Lambda_{\lambda}$ commutes with $\left.C D C^{-1}\right)$ gives $E+\sum_{i=1}^{n-3} E_{i n}+2 E_{(n-2) n}$. Then multiplying by $\Lambda_{n-3}^{2}$ gives $E+\sum_{i=1}^{n-4} E_{i n}+3 E_{(n-3) n}$. Continuing in this manner finally gives the matrix $E+(n-1) E_{1 n}$. Formally, 


$$
\left(\prod_{j=1}^{n-2} \Lambda_{(n-1)-j}^{j}\right)\left(C D C^{-1}\right)=E+(n-1) E_{1 n}
$$

Since $p$ does not divide $n-1$, there is an integer $t$ such that $t(n-$ 1) $\equiv 1 \bmod p$. Then

$$
\left(E+(n-1) E_{1 n}\right)^{t}=E+E_{1 n} .
$$

This shows that $\operatorname{sgp}\{C, D\}$ contains the transvection $E+E_{1 n}$, and completes the proof of Theorem 3 .

Acknowledgement. I thank Dr. M. F. Newman for his help and encouragement.

\section{REFERENCES}

1. H. S. M. Coxeter and W. O. J. Moser, Generators and Relations for Discrete Groups, 2nd edition, Ergebnisse der Mathematik, Band 14, Springer-Verlag, Berlin-GöttingenHeidelberg-New York, 1965.

2. Yu. M. Gorčakov and V. M. Levčuk, Concerning a residual property of free groups, Algebra i Logika, 9 (1970), 415-421 (Russian).

3. Hermann Heineken and Peter M. Neumann, Identical relations and decision procedures for groups, J. Austral. Math. Soc., 7 (1967), 39-47.

4. Nathan Jacobson, Lectures in Abstract Algebra, Volume 1, van Nostrand, Princeton, 1951.

5. Robert A. Katz and Wilhelm Magnus, Residual properties of free groups, Comm. Pure Appl. Math., 22 (1969), 1-13.

6. Ada Peluso, A residual property of free groups, Comm. Pure Appl. Math., 19 (1966), 435-437.

7. Samuel Poss, A residual property of free groups, Comm. Pure Appl. Math., 23 (1970), 749-756.

8. Joseph J. Rotman, The Theory of Groups: An Introduction, Allyn and Bacon, Boston, 1965.

Received September 23, 1971. I would like to acknowledge the support of an Australian Commonwealth Postgraduate Award.

Institute of AdVANCEd Studies

AUstralian National UNIVERSity

CANberra, ACT. 



\section{PACIFIC JOURNAL OF MATHEMATICS}

\section{EDITORS}

\author{
H. SAMELSON \\ Stanford University \\ Stanford, California 94305 \\ C. R. HOBBY \\ University of Washington \\ Seattle, Washington 98105
}

\section{J. DugundjI}

Department of Mathematics University of Southern California Los Angeles, California 90007

\section{RICHARD ARENS}

University of California

Los Angeles, California 90024

\section{ASSOCIATE EDITORS}
E. F. BECKENBACH
B. H. NeumanN
F. WOLF
K. YosHIDA

\section{SUPPORTING INSTITUTIONS}

\author{
UNIVERSITY OF BRITISH COLUMBIA \\ CALIFORNIA INSTITUTE OF TECHNOLOGY \\ UNIVERSITY OF CALIFORNIA \\ MONTANA STATE UNIVERSITY \\ UNIVERSITY OF NEVADA \\ NEW MEXICO STATE UNIVERSITY \\ OREGON STATE UNIVERSITY \\ UNIVERSITY OF OREGON \\ OSAKA UNIVERSITY
}

\author{
UNIVERSITY OF SOUTHERN CALIFORNIA \\ STANFORD UNIVERSITY \\ UNIVERSITY OF TOKYO \\ UNIVERSITY OF UTAH \\ WASHINGTON STATE UNIVERSITY \\ UNIVERSITY OF WASHINGTON \\ ${ }^{*} \stackrel{*}{*}{ }^{*}$
AMERICAN MATHEMATICAL SOCIETY
NAVAL WEAPONS CENTER
}

The Supporting Institutions listed above contribute to the cost of publication of this Journal, but they are not owners or publishers and have no responsibility for its content or policies.

Mathematical papers intended for publication in the Pacific Journal of Mathematics should be in typed form or offset-reproduced, (not dittoed), double spaced with large margins. Underline Greek letters in red, German in green, and script in blue. The first paragraph or two must be capable of being used separately as a synopsis of the entire paper. The editorial "we" must not be used in the synopsis, and items of the bibliography should not be cited there unless absolutely necessary, in which case they must be identified by author and Journal, rather than by item number. Manuscripts, in duplicate if possible, may be sent to any one of the four editors. Please classify according to the scheme of Math. Rev. Index to Vol. 39. All other communications to the editors should be addressed to the managing editor, Richard Arens, University of California, Los Angeles, California, 90024.

50 reprints are provided free for each article; additional copies may be obtained at cost in multiples of 50 .

The Pacific Journal of Mathematics is published monthly. Effective with Volume 16 the price per volume (3 numbers) is $\$ 8.00$; single issues, $\$ 3.00$. Special price for current issues to individual faculty members of supporting institutions and to individual members of the American Mathematical Society: $\$ 4.00$ per volume; single issues $\$ 1.50$. Back numbers are available.

Subscriptions, orders for back numbers, and changes of address should be sent to Pacific Journal of Mathematics, 103 Highland Boulevard, Berke'ey, California, 94708.

PUBLISHED BY PACIFIC JOURNAL OF MATHEMATICS, A NON-PROFIT CORPORATION

Printed at Kokusai Bunken Insatsusha (International Academic Printing Co., Ltd.), 270, 3-chome Totsuka-cho, Shinjuku-ku, Tokyo 160, Japan. 


\section{Pacific Journal of Mathematics}

\section{Vol. 43, No. $3 \quad$ May, 1972}

Max K. Agoston, An obstruction to finding a fixed point free map on a manifold.... 543

Nadim A. Assad and William A. Kirk, Fixed point theorems for set-valued mappings

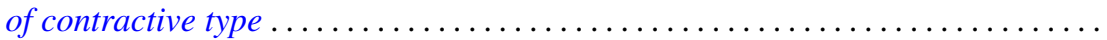

John Winston Bunce, Characterizations of amenable and strongly amenable

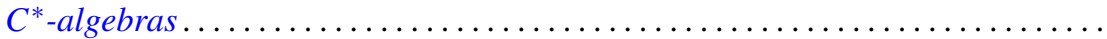

Erik Maurice Ellentuck and Alfred Berry Manaster, The decidability of a class of

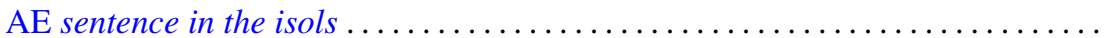

U. Haussmann, The inversion theorem and Plancherel's theorem in a Banach

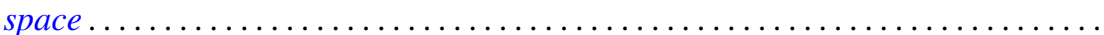

Peter Lawrence Falb and U. Haussmann, Bochner's theorem in infinite dimensions.

Peter Fletcher and William Lindgren, Quasi-uniformities with a transitive base ..... Dennis Garbanati and Robert Charles Thompson, Classes of unimodular abelian

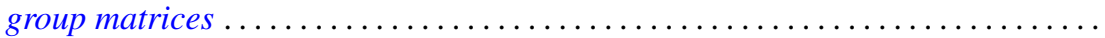

Kenneth Hardy and R. Grant Woods, On c-realcompact spaces and locally bounded normal functions

Manfred Knebusch, Alex I. Rosenberg and Roger P. Ware, Grothendieck and Witt rings of hermitian forms over Dedekind rings .......................

George M. Lewis, Cut loci of points at infinity.

Jerome Irving Malitz and William Nelson Reinhardt, A complete countable $L_{\omega_{1}}^{Q}$ theory with maximal models of many cardinalities . . . . . . . . . . . . . . . . .

Wilfred Dennis Pepe and William P. Ziemer, Slices, multiplicity, and Lebesgue

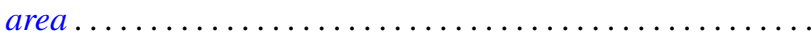

Keith Pierce, Amalgamating abelian ordered groups . .

Stephen James Pride, Residual properties of free groups . . . . . . . . . . . . . 725

Roy Martin Rakestraw, The convex cone of n-monotone functions .

T. Schwartzbauer, Entropy and approximation of measure preserving transformations .

Peter F. Stebe, Invariant functions of an iterative process for maximization of a polynomial...

Kondagunta Sundaresan and Wojbor Woyczynski, L-orthogonally scattered

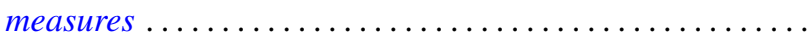

Kyle David Wallace, $C_{\lambda}$-groups and $\lambda$-basic subgroups $\ldots \ldots \ldots$

Barnet Mordecai Weinstock, Approximation by holomorphic functions on certain

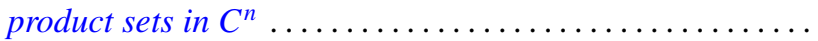

Donald Steven Passman, Corrections to: "Isomorphic groups and group rings”.

Don David Porter, Correction to: "Symplectic bordism, Stiefel-Whitney numbers, and a Novikov resolution"

John Ben Butler, Jr., Correction to: “Almost smooth perturbations of self-adjoint operators".

Constantine G. Lascarides, Correction to: "A study of certain sequence spaces of Maddox and a generalization of a theorem of Iyer" ...... ...

George A. Elliott, Correction to: "An extension of some results of takesaki in the reduction theory of von neumann algebras" ......................... 